\title{
Partial prostate re-irradiation for the treatment of isolated local recurrence of prostate cancer in patients previously treated with primary external beam radiotherapy: short-term results of a monocentric study
}

\author{
Fabio MATRONE ${ }^{1, *}$, Alberto REVELANT ${ }^{1}$, Giuseppe FANETTI ${ }^{1}$, Jerry POLESEL ${ }^{2}$, Paola CHIOVATI ${ }^{3}$, Michele AVANZO , Filippo DE RENZI $^{4}$, \\ Carlotta Benedetta COLOMBO ${ }^{5}$, Mauro ARCICASA ${ }^{1}$, Antonino DE PAOLI ${ }^{1}$, Giovanni FRANCHIN ${ }^{1}$, Roberto BORTOLUS ${ }^{1}$
}

\begin{abstract}
${ }^{1}$ Department of Radiation Oncology, Centro di Riferimento Oncologico di Aviano (CRO) IRCCS, Aviano, Italy; ${ }^{2}$ Unit of Cancer Epidemiology, Centro di Riferimento Oncologico di Aviano (CRO) IRCCS, Aviano, Italy; ${ }^{3}$ Department of Medical Physics, Centro di Riferimento Oncologico di Aviano (CRO) IRCCS, Aviano, Italy; ${ }^{4}$ Radiotherapy Unit, San Martino Hospital, Belluno, Italy; ${ }^{5}$ Department of Nuclear Medicine, Centro di Riferimento Oncologico di Aviano (CRO) IRCCS, Aviano, Italy
\end{abstract}

*Correspondence: fabio.matrone@cro.it

Received June 22, 2020 / Accepted September 23, 2020

\begin{abstract}
Many different therapeutic options are available for locally recurrent prostate cancer (PCa). However, standard treatment has not yet been established. We conducted a partial prostate re-irradiation (PPR) program for the treatment of isolated and limited-size intraprostatic recurrences, in patients who previously underwent external beam radiation therapy (EBRT) as primary treatment for prostatic cancer (PCa). The analysis of this experience in terms of feasibility, toxicity, and efficacy is reported. The inclusion criteria of this retrospective analysis were: previous definitive EBRT, evidence of biochemical recurrence, radiological detection of isolated local relapse, and PPR as local salvage therapy. Gastrointestinal (GI) and genitourinary (GU) toxicities were registered according to the RTOG/EORTC criteria. Between July 2012 and May 2019, 44 patients were treated with PPR. All patients completed the planned treatment. The median follow-up was 25.4 months. Tumor progression was observed in 18 patients (40.9\%). Two-year local control, biochemical failure-, and clinical relapse-free survival rates were $90.1 \%, 58.3 \%$, and $67.9 \%$, respectively. The occurrence of biochemical failure after PPR is lower for patients with the time interval between the primary EBRT and first biochemical failure $>4$ years; local control results strongly associated with a biologically effective dose (BED) at first EBRT >177 Gy. No acute grade 3 or greater toxic events were observed. Two late grade $3 \mathrm{GU}$ toxicities were reported. Although retrospective in design, our study indicates that PPR appears as a feasible, well-tolerated, and effective salvage treatment for isolated local PCa recurrence. Long term data are required in order to confirm these results.
\end{abstract}

Key words: prostate cancer, salvage therapy, recurrence, re-irradiation

External beam radiotherapy (EBRT) and surgery represent a therapeutic milestone in the treatment of localized prostate cancer (PCa) [1]. In recent years, advances in radiation therapy techniques have resulted in a significant improvement of both tumor control and toxicity associated with treatment $[2,3]$. However, about half of prostate cancer patients undergoing EBRT as their primary treatment develop a biochemical disease recurrence within ten years $[4,5]$. At imaging re-evaluation, a percentage ranging from $30-60 \%$ of these patients show a recurrence limited to the prostate gland [6, 7].

Historically, the most common treatment choice for locally recurrent $\mathrm{PCa}$ was androgen deprivation therapy (ADT). However, a downside of this approach is its detrimental effect on the quality of life as well as the risk of promoting a castration-resistant disease. Nowadays, international guidelines suggest a local approach (i.e. salvage prostatectomy, re-irradiation with brachytherapy or EBRT, cryotherapy, and high intensity focused ultrasound) to manage this clinical picture. However, the most effective strategy for recurrent PCa remains still unknown. Additionally, no agreement has been reached so far as to which is the optimal patient selection for each local approach [8].

In recent years, there has been a growing interest in re-irradiation, due to the introduction of advanced radiation therapy techniques (i.e. Stereotactic Body Radiotherapy or SBRT), which allow to administer ablative doses to small volumes, thus sparing adjacent healthy tissues [9]. 
At the same time, advancements in radiological and nuclear medicine fields, with the development and improvement of multiparametric Magnetic Resonance Imaging (MRI) and Positron Emission Computed Tomography (PET-CT) with choline and the most recent Prostate Specific Membrane Antigen (PSMA), respectively, have improved the capacity to detect with greater accuracy the site of disease recurrence and its local extension [10]. These technological and diagnostic advancements have had a significant impact on the radiation therapy field and they have also contributed to the idea of limiting re-irradiation to intraprostatic recurrence only.

Irradiation of intraprostatic recurrence only has the two-fold aim of limiting the irradiation volume, thus allowing the delivery of ablative doses to the true lesion, and of reducing the dose to the previously irradiated adjacent areas, with a favorable impact on treatment-related toxicity.

To date, few studies have been reported in the literature on Partial Prostate Re-irradiation (PPR); however, these have been conducted on heterogeneous patient populations by primary treatment or by volume of re-irradiation, with most patients receiving re-treatment to the entire prostatic gland and a minority to the site of recurrence only.

Based on these premises, we conducted a retrospective analysis on PPR for the treatment of isolated and limitedsize intraprostatic recurrences, in patients who previously underwent EBRT as primary treatment for prostatic cancer. The goal of the present study is to evaluate PPR in terms of feasibility, toxicity, and efficacy.

\section{Patients and methods}

Patients' selection. We retrospectively reviewed the medical records of 51 consecutive patients treated with PPR between July 2012 and May 2019 for isolated local recurrence of PCa after radical EBRT at the Centro di Riferimento Oncologico di Aviano (CRO) IRCCS, Aviano, Italy.

For all patients, a biochemical recurrence (BCR) according to Phoenix criteria (PSA nadir $+2 \mathrm{ng} / \mathrm{ml}$ ) was recorded [11]. Subsequent imaging investigations included pelvic and prostatic MRI (T2-weighted-T2W, diffusionweighted-DWI, and dynamic contrast-enhanced-DCE) and/ or [11C]-choline PET-CT. Patients were eligible to PPR only if they met the following criteria: diagnosis of local recurrence 12 months or more after primary EBRT, Karnofsky performance status $>80$, absence or minimal gastrointestinal and genitourinary late effects after the primary irradiation, imaging documenting an isolated intraglandular recurrence occupying less than $50 \%$ of the prostate. Prostate biopsy was not mandatory if all diagnostic findings were univocal in presence of a body of evidence (PSA kinetic, prostate MRI, and/or [11C]-choline PET-CT findings) in favor of a local recurrence. Four patients were excluded due to metastatic disease (oligometastatic) at the time of re-irradiation, one patient was excluded because of another local salvage treatment between first EBRT and PPR, and two patients because of a follow-up shorter than 6 months, thus leaving 44 eligible patients. All patients included in this study had been evaluated at the time of recurrence by a multidisciplinary tumor board and signed written informed consent for PPR.

The study was approved and retrospectively registered by the Institutional Review Board and by the Ethics Committee (Comitato Etico Unico Regionale) (study registration number: CRO 2020-14).

PPR protocol. PPR was delivered by Linac-based external beam radiation. All patients received a total dose of $35 \mathrm{~Gy}$ in seven daily fractions of $5 \mathrm{~Gy}$, five fractions/week, which translates into a biologically effective dose (BED) of $151.7 \mathrm{~Gy}$ (for $\alpha / \beta 1.5 \mathrm{~Gy}$ ). No rectal spacer or fiducial markers were used. Patients underwent a non-contrast-enhanced multislice CT scan with a $2 \mathrm{~mm}$ slice thickness. Patient immobilization during $\mathrm{CT}$ acquisition and treatment was obtained by knee-fix ${ }^{\circledast}$ and feet-fix ${ }^{\oplus}$ (CIVCO Medical Solutions, Kalona, IA, USA). All patients were asked to empty the bowel (oral and written instructions for diet and enema were given) and to have full urinary bladder for computed tomography (CT) planning and during all treatment fractions (patients were educated to drink half a liter of water 30 minutes before the procedure). PCa local recurrence was detected by MRI (as the area showing low signal intensity on $\mathrm{T} 2 \mathrm{~W}+$ focal hypointensity on the ADC map and hyperintensity on high b-value imaging on DWI + hyperenhancement on DCE) [12] and/ or by [11C]-choline PET-CT (as the area delineated with a semiautomated technique using a fixed threshold of $40 \%$ of the maximum signal intensity) [13]. This area was defined as the gross tumor volume (GTV). MRI and/or [11C]-choline PET-CT images fusion based on rigid transformations (Registration; Varian Medical Systems, Palo Alto, CA) was used to delineate GTV on planning CT. Clinical Target Volume (CTV) was defined as the GTV plus $3 \mathrm{~mm} \mathrm{[14].}$ The Planning Target Volume (PTV) included the CTV and a margin of $3 \mathrm{~mm}$ [15]. On each slice, contouring of organs at risk was also performed: rectum, bladder, penile bulb, and femoral heads. The planning objectives for the target were the following: at least $98 \%$ of the CTV to be covered by $98 \%$ of the prescribed dose (V98\% >98\%) and at least $99 \%$ of the PTV to be covered by $95 \%$ of the dose (V95\% >99\%). Hot spots $>107 \%$ were accepted only if inside the CTV. Patients were treated by Linac Volumetric Modulated Arc Therapy (VMAT) technique (RapidArc ${ }^{\oplus}$, Varian Medical Systems, Palo Alto, CA) with 2 arcs. In particular, in this study all treatment plans were optimized and delivered on a Trilogy or a TrueBeam ${ }^{\bullet}$ Linac (Varian Medical Systems, Palo Alto, CA, USA), with 18-MV or 10-MV flattening-filter-free photon beams, respectively; the maximum dose rate available was $600 \mathrm{MU} / \mathrm{min}$ and $2400 \mathrm{MU} / \mathrm{min}$, respectively. The treatment planning system in use was Eclipse version 13.6 with Anisotropic Analytical Algorithm version 13.6.23 (Varian Medical System, Palo Alto, CA, USA) with a grid resolution of $2.5 \mathrm{~mm}$ or less. Avoidance sectors were planned in the case of metal implants in the femoral heads of patients. Dose-volume 
objectives applied during plan optimization for organs at risk (OARs) were as follows: for rectum, V10 Gy $<40 \%$, V18 Gy $<20 \%$, V26 Gy $<10 \%$; for bladder, V10 Gy $<25 \%$, V18 Gy $<15 \%$, V26 Gy $<10 \%$; for femoral heads, V10 $<1 \%$; for penile bulb, V10 <5\%. Image guidance was performed before each fraction. In particular, before each fraction, a cone beam CT (CBCT) was performed to verify the correct position as well as full bladder and empty rectum. Set up corrections were performed after automatic matching of CBCT images to reference planning CT (using the integrated algorithm on the Varian on-board imager console) followed by manual adjustments with an action level of $1 \mathrm{~mm}$. When set up corrections were greater than $5 \mathrm{~mm}$, or bladder and rectum preparation was not correct, the patient was repositioned and re-verified. The entire process, starting from the CBCT acquisition to the end of radiation delivery, always took less than 6 minutes.

Short-term ADT with luteinizing hormone-releasing hormone agonist (LHRHa) was prescribed in the case of PSA doubling time $<12$ months.

Follow-up after PPR. After PPR, PSA level dosage was recorded at 3 months after treatment, every 3 months for the following 2 years, every 6 months until the fifth year, then annually. Likewise, the primary treatment, BCR after PPR was defined as PSA nadir $+2 \mathrm{ng} \cdot \mathrm{ml}^{-1}$ above the nadir according to Phoenix Consensus criteria. In the case of BCR, a further [11C]-choline-PET-CT was performed to define local, regional, or metastatic failure. Depending on the type of disease progression, patients received additional therapies. The vital status, the date, and the underlying cause of death (i.e., the condition that led to death) were ascertained up to December 31 $1^{\text {st }}, 2019$.

Acute ( $<3$ months) and late ( $>3$ months) toxicities were registered according to the Radiation Therapy Oncology Group/European Organization for Research and Treatment of Cancer (RTOG/EORTC) criteria during PPR, and subsequently every 3-6 months up to the fifth year from PPR [16]. Gastrointestinal (GI) and genitourinary (GU) events were registered. Sexual dysfunction was not analyzed due to the lack of baseline evaluation.

Statistical analysis. For each patient, the time at risk was computed as the time elapsed from the end of PPR to the occurrence of the events of interest which were: PSA increase (defined as above) for biochemical failure-free survival (BFFS); radiological detection of local or distant disease for clinical relapse-free survival (CRFS); radiological diagnosis of prostatic recurrence for local control (LC); radiological diagnosis of distant locoregional or metastatic disease for distant relapse-free survival (DRFS); death for overall survival (OS).

The survival probabilities were estimated by means of the Kaplan-Meier method and survival differences were tested through the log-rank test [17]. Differences in clinical outcomes (i.e., OS, BFFS, CRFS, LC, DRFS) according to potential predictors were evaluated through the log-rank test [17]. Statistical significance was claimed for $\mathrm{p}<0.05$ (two-tailed).

\section{Results}

Patient and tumor characteristics. Patients characteristics are shown in Table 1. At primary EBRT, our population was mainly constituted by patients initially presenting with high-risk disease (23 patients, 52.3\%) according to D’Amico classification [18]; low-risk and intermediate-risk disease was reported in $7(15.9 \%)$ and $14(31.8 \%)$ patients, respec-

Table 1. Clinical characteristics of 44 prostate cancer patients with isolated recurrence.

\begin{tabular}{|c|c|}
\hline Characteristics & $\mathbf{n}$ \\
\hline \multicolumn{2}{|l|}{ Prior RT } \\
\hline Age at diagnosis [years], median (range) & $68(51-81)$ \\
\hline Initial PSA [ng.ml-1], median (range) & $8.7(2.6-46)$ \\
\hline Initial Gleason Score, median (range) & $7 a(6-9)$ \\
\hline \multicolumn{2}{|l|}{ Prior RT modality } \\
\hline 3DCRT & $28(64 \%)$ \\
\hline IMRT & $16(36 \%)$ \\
\hline $\mathrm{BED}^{\star}(\mathrm{Gy})$, median (range) & $177.3(151.7-186.7)$ \\
\hline Number of fractions, median (range) & $37(27-42)$ \\
\hline Prophylactic pelvic irradiation & $2(4 \%)$ \\
\hline $\begin{array}{l}\text { Interval between first RT and BCR [months], } \\
\text { median (range) }\end{array}$ & $60(16.9-615.5)$ \\
\hline \multicolumn{2}{|l|}{ PPR } \\
\hline Age at PPR [years], median (range) & $76(56-89)$ \\
\hline Pre-PPR PSA [ng.ml-1 ${ }^{-1}$, median (range) & $2.6(2-7.68)$ \\
\hline \multicolumn{2}{|l|}{ Concomitant androgen deprivation to PPR } \\
\hline Yes $(\%)$ & $12(27 \%)$ \\
\hline No $(\%)$ & $32(73 \%)$ \\
\hline Duration [months], median (range) & $4.2(2.9-7.1)$ \\
\hline \multicolumn{2}{|l|}{ Biopsy of the target lesion } \\
\hline Yes $(\%)$ & $5(11 \%)$ \\
\hline Gleason score (range) & $6(6-7 a)$ \\
\hline No $(\%)$ & $39(89 \%)$ \\
\hline \multicolumn{2}{|l|}{ Histological +/-Radiological diagnosis } \\
\hline Biopsy + PET-CT + MRI & 1 \\
\hline Biopsy + MRI & 2 \\
\hline Biopsy + PET-CT & 2 \\
\hline PET-CT only & 27 \\
\hline PET-CT + MRI & 2 \\
\hline MRI only & 10 \\
\hline \multicolumn{2}{|l|}{ Target lesion } \\
\hline left lobe & 15 \\
\hline right lobe & 16 \\
\hline apex & 2 \\
\hline bilateral anteriorly & 2 \\
\hline bilateral posteriorly & 9 \\
\hline
\end{tabular}

${ }^{\star}$ BED was calculated for $\alpha / \beta$ 1.5 Gy; Abbreviations: 3DCRT-Conformal radiotherapy; $\mathrm{BCR}$-Biochemical recurrence; $\mathrm{BED}$-Biologically effective dose; IMRT-Intensity modulated radiotherapy; MRI-Magnetic Resonance Imaging; PET-CT Positron Emission Computed Tomography; PPR-Partial Prostate Re-irradiation; PSA-Prostate specific antigen; RT-Radiotherapy 
tively. The first EBRT included 3D-CRT in 28 (64\%) patients (median BED: $177.3 \mathrm{~Gy}$; the median number of fractions: 38 ) and IMRT in 16 (36\%) (median BED: $180.3 \mathrm{~Gy}$; the median number of fractions: 28). Twenty-seven (61\%) patients were treated with conventional fractionation, and 17 (39\%) received moderate hypofractionation. In the study population, BED of first EBRT was not associated with D'Amico risk classification (data not shown).

According to risk group evaluation, concurrent ADT was added to primary radiation therapy in 37 (84\%) patients.

The median time between first EBRT and BCR was 81.1 months, 67.3 months, and 54 months in low-risk, intermediate-risk, and high-risk disease patients, respectively.

Table 2. Dosimetric parameters in 44 patients receiving Partial Prostatic Re-irradiation.

\begin{tabular}{lcccc}
\hline Dosimetric parameter & Median & Mean & Q1 & Q3 \\
\hline CTV $\left(\mathrm{cm}^{3}\right)$ & 16 & 16.3 & 6 & 23 \\
PTV $\left(\mathrm{cm}^{3}\right)$ & 26 & 27.9 & 15.1 & 37.5 \\
Maximum bladder dose $(\mathrm{Gy})$ & 34.4 & 29.4 & 28.8 & 36.2 \\
Mean bladder $(\mathrm{Gy})$ & 2.7 & 6.4 & 2 & 10.2 \\
Bladder V90\% $\left(\mathrm{cm}^{3}\right)$ & 0.1 & 1.3 & 0 & 2.4 \\
Bladder V100\% $\left(\mathrm{cm}^{3}\right)$ & 0 & 0.5 & 0 & 0.5 \\
Maximum rectal dose $(\mathrm{Gy})$ & 35.4 & 34.3 & 33.8 & 35.6 \\
Mean rectal dose $(\mathrm{Gy})$ & 6.6 & 7.4 & 5.6 & 9.3 \\
Rectal V90\% $\left(\mathrm{cm}^{3}\right)$ & 0.3 & 0.5 & 0 & 0.8 \\
Rectal V100\% $\left(\mathrm{cm}^{3}\right)$ & 0 & 0.1 & 0 & 0 \\
\hline Abbreviations: $\mathrm{CTV}^{3} \mathrm{Clinical}$ & & & & 0.3 \\
\hline
\end{tabular}

Abbreviations: CTV-Clinical target volume; PTV-Planning target volume; Q1-First quartile; Q3-Third quartile; V90\%-Volume receiving 90\% of the prescribed dose; V100\%-Volume receiving $100 \%$ of the prescribed dose

Table 3. Description of 18 relapses in prostate cancer patients undergoing Partial Prostate Re-irradiation.

\begin{tabular}{lc}
\hline Outcome & n (\%) \\
\hline Biochemical relapse only & $4(22 \%)$ \\
Clinical recurrence & \\
Local & $4(22 \%)$ \\
Local and distant & $3(17 \%)$ \\
Distant & $7(39 \%)$ \\
Locoregional nodes and metastatic sites & $1(6 \%)$ \\
Locoregional nodes & $5(28 \%)$ \\
Metastatic sites & $1(6 \%)$ \\
\hline
\end{tabular}

Table 4. Toxicities in 44 prostate cancer patients undergoing Partial Prostate Re-irradiation (PPR).

\begin{tabular}{lcclccc}
\hline \multicolumn{3}{c}{ Acute toxicity } & & \multicolumn{3}{c}{ Late toxicity } \\
\cline { 1 - 3 } \cline { 5 - 6 } Grade & GU & GI & & Grade & GU & GI \\
\hline G0 & $30(68 \%)$ & $40(92 \%)$ & G0 & $28(64 \%)$ & $41(93 \%)$ \\
G1 & $10(23 \%)$ & $2(4 \%)$ & & G1 & $8(18 \%)$ & $3(7 \%)$ \\
G2 & $4(9 \%)$ & $2(4 \%)$ & G2 & $6(14 \%)$ & 0 \\
G3 & 0 & 0 & & G3 & $2(4 \%)$ & 0 \\
Total & & & Total & & \\
patients & 44 & 44 & patients & 44 & 44 \\
\hline Abbreviations: GU-Genitourinary; GI-Gastrointestinal
\end{tabular}

Prostatic recurrence was documented by [11C]-choline PET-CT, pelvic MRI, or both in 29 (66\%), 12 (27\%), and $3(7 \%)$ patients, respectively. Biopsy of the radiologically documented recurrent lesion was performed in $5(11 \%)$ patients. For the remaining 39 (89\%) patients, the diagnosis of isolated local recurrence of prostate cancer was based on PSA levels indicating a BCR and on the radiological confirmation. In most cases, local recurrence had clear laterality: it occurred within the right lobe in $16(36 \%)$ patients and within the left lobe in 15 (34\%) patients, respectively.

Concomitant ADT was used in $12(27 \%)$ patients for a median time of 4.2 months (2.9-7.1 months). The median PTV volume was $26 \mathrm{~cm}^{3}\left(5.7-56 \mathrm{~cm}^{3}\right)$. Dosimetry details of target volumes and organs at risk are available in Table 2.

Tumor outcome. All patients completed the planned treatment. The median PSA nadir after PPR was $1.1 \mathrm{ng} \cdot \mathrm{ml}^{-1}$ (0.07-4.92 ng. $\left.\mathrm{ml}^{-1}\right)$. Details of the post-PPR progressive disease are summarized in Table 3.

After a median follow-up of 25.4 months (6.7-81.5 months), biochemical failure was observed in 18 (41\%) patients with a median time to relapse of 16.8 months (7.6-53 months). In all cases, clinical relapse was preceded by biochemical failure.

At restaging, in $7(39 \%)$ out the 18 patients, post-PPR recurrence was located in the prostate (four patients in the prostate only, 2 in prostate and locoregional nodes, 1 in prostate and bone metastases); all prostatic recurrences were inside the treatment field.

The 1- and 2-year actuarial rates were $85.9 \%$ and $58.3 \%$ for BFFS, $85.9 \%$ and $67.9 \%$ for CRFS, $94.4 \%$ and $90.1 \%$ for LC, and $88.4 \%$ and $73.8 \%$ for DRFS, respectively. No patient died during the first two years after PPR (OS $=100 \%)$.

At the last follow-up, 43 (98\%) patients were alive, 26 (59\%) patients showed no evidence of disease, and 17 (39\%) were alive with biochemical or clinical disease. One (2\%) patient with clinical recurrence died of other causes. Metastatic disease was reported in 4 (9\%) patients, 2 of them developed metastatic castration-resistant prostate cancer (mCRPC).

Late recurrence (i.e., the time interval between primary EBRT and first BCR $>4$ years) was a favorable prognostic factor for BFFS $(p=0.046)$, with a 5 -year rate of $67 \%$ in patients with late recurrence versus $0 \%$ in patients with early recurrence (i.e., the time interval between primary EBRT and first BCR $<4$ years; Figure 1A). Similarly, patients with BED of the first radiotherapy $>177$ Gy had a 5-year BFFS rate of $60 \%$ compared to $0 \%$ in patients with $\mathrm{BED}<177 \mathrm{~Gy}$ $(p=0.071$; Figure $1 B)$. Furthermore, BED of the first radiotherapy was strongly correlated with LC, and no patient receiving a $\mathrm{BED}>177 \mathrm{~Gy}$ experienced a local recurrence $(p<0.001$; Figure 1C); whereas there was no statistically significant association between BED >177 Gy and DRFS $(p=0.267$; Figure 1D). The radiation dose of primary EBRT was converted into BED using $\alpha / \beta=1.5 \mathrm{~Gy}$ : since there is no consensus on the value of $\alpha / \beta$ for prostate cancer, as a sensitivity analysis on $\alpha / \beta$, analyses were performed with different 

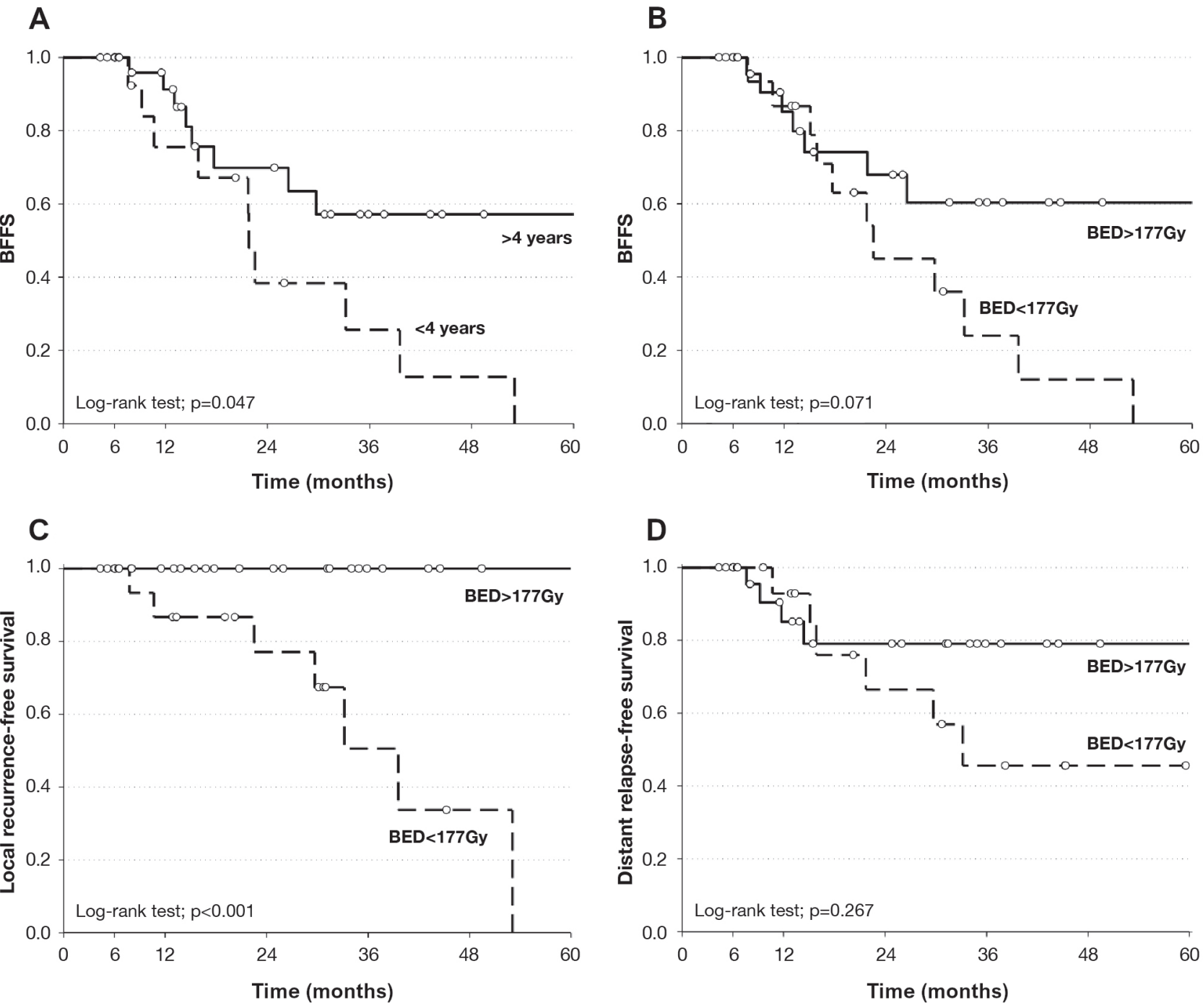

Figure 1. Biochemical failure-free survival according to time to first recurrence (A) and BED (B); local recurrence-free survival (C) and distant relapsefree survival (D) according to BED. Abbreviations: BFFS-Biochemical failure-free survival; BED-Biologically effective dose

values of $\alpha / \beta$ (2 Gy, $3.5 \mathrm{~Gy}, 5 \mathrm{~Gy})$ [19], showing no substantial differences (data not shown).

Patients undergoing ADT concomitant to PPR reported a better BFFS than those who did not receive ADT (5-year BFFS: $45 \%$ and $16 \%$, respectively), but the difference was not statistically significant $(\mathrm{p}=0.119)$. Similarly, non-significant effect of concomitant ADT was found for CRFS ( $\mathrm{p}=0.197)$, LC $(\mathrm{p}=0.276)$, and DRFS $(\mathrm{p}=0.191)$.

Toxicity. Overall acute toxicities were assessed in 17 (39\%) patients (Table 4).

Considering the maximum grade of toxicity observed up to 3 months after PPR, 27 (61\%) patients had no acute toxicities. No patient experienced acute Grade 3 GU or GI toxicity. Two (5\%) patients experienced a late Grade 3 GU event consisting of frequent macroscopic hematuria requiring blood transfusion. No patient developed late Grade 3 GI or Grade $4-5$ GU or GI toxicities.

In 13 (30\% of our series) patients monitored for more than 36 months after PPR, only two Grade $\geq 2$ GU/GI events were reported (one GU Grade 3 and one GU Grade 2). We found no association between toxicities PPR-related and BED of the first radiotherapy, the time interval between initial EBRT and $\mathrm{PPR}$, and re-treatment volumes.

\section{Discussion}

In patients with isolated local recurrence of $\mathrm{PCa}$, local salvage therapies could represent a valid alternative to ADT, because they may offer, unlike hormone therapy, curative prospects. On the other hand, since local salvage therapies 


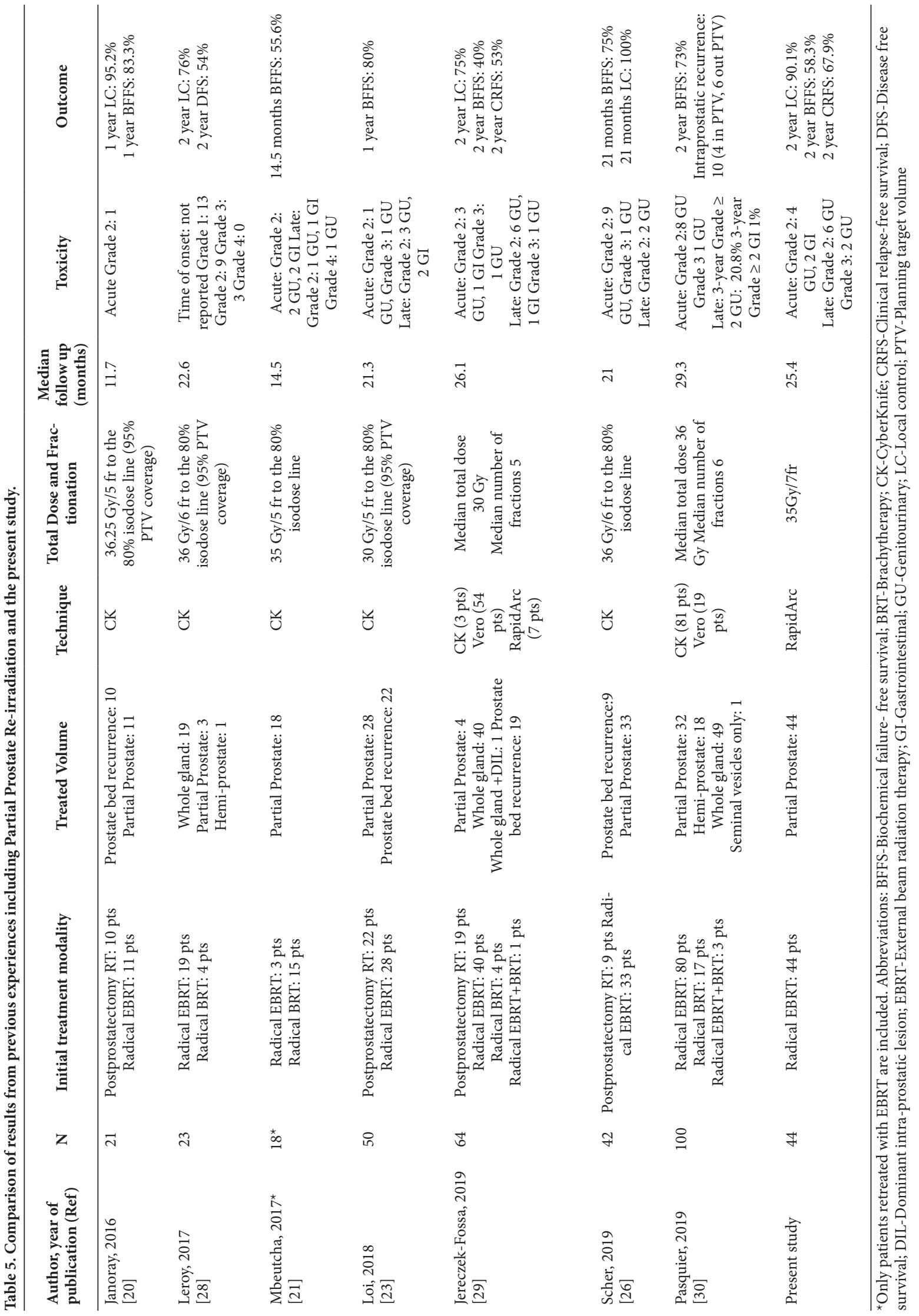


act on already treated tissues (surgically or with radiation therapy), it is a priority that any local treatment option should guarantee an acceptable profile of toxicity. Among the spectrum of local salvage therapies available, PPR could represent a valid approach. In this retrospective study, we focused on PPR with the aim of evaluating its efficacy, feasibility, and toxicity profile.

Several investigations evaluating the efficacy of salvage re-irradiation for locally recurrent PCa were published [20-30]; however, the comparability of our results with other reports is hindered by the different selection criteria adopted by the authors. Some papers included in the same case series patients with local recurrence of PCa after primary EBRT, after BRT, or after salvage post-prostatectomy radiotherapy [20-30]. In addition, investigations evaluating intraprostatic recurrence after primary EBRT reported re-irradiation with mixed approaches delivering radiation therapy to the entire prostatic gland or to hemi prostate or to part of the prostate [28-30]. In our retrospective cohort, we included only patients who underwent EBRT as primary treatment and who were subsequently treated with PPR for an isolated intraprostatic recurrence.

Our results in terms of BFFS and LC rates are in agreement with updated literature (Table 5).

Janoray et al. [20] using a CyberKnife ${ }^{\circledast}$ linac and a total dose of $36.25 \mathrm{~Gy}$ in five fractions, treated a population of 21 patients with biochemical failure with focal salvage radiotherapy. Eleven of these patients had been previously treated with radical EBRT with a 1-year biochemical response rate and local control of $83.3 \%$ and $95.2 \%$, respectively.

Similarly, Mbeutcha et al. [21] analyzed a total population of 28 patients with local recurrence after definitive EBRT. Eighteen of these patients underwent focal radiotherapy using CyberKnife ${ }^{\circledast}$ linac and received a total dose of 35 Gy in five daily fractions. Within a median follow-up of 14.5 months, 10 out of 18 patients remained alive with no evidence of disease; among the patients who experienced BCR, only 3 (20.0\%) developed a loco-regional disease recurrence.

The largest series on salvage re-irradiation has been reported in a retrospective multicenter study recently published by Pasquier et al. [30]. On a total of 100 cases of local relapse, 32 were treated with PPR with different radiation doses. The authors reported excellent outcomes with a 2 -year overall BFFS of $73 \%$ and only four in-field recurrences.

In our study, the toxicity profile after PPR resulted highly favorable, with a very low rate of GU and GI side effects. In fact, none of our patients discontinued PPR for acute toxicity and only two Grade 3 late toxicity events were reported (equivalent to $4 \%$ of the total patient population). No Grade $\geq 4$ toxicity was recorded. Our data are in agreement with those reported in the literature; however, our median followup, although similar to those reported in most investigations dedicated to PCa re-irradiation, might be too short for an accurate estimate of late toxicity.
Our results on late toxicity differ greatly from those published by Zilli et al. [25] who, in a series of 14 patients treated with whole prostate re-irradiation, at a longer median follow-up of 94 months, reported a high rate of both late GU and GI toxicities (eight GU Grade $\geq 3$ toxicity events and nine Grade $\geq 3$ GI events). It should be noted that in the series by Zilli et al. conformal radiotherapy (3DCRT) + brachytherapy boost was the modality predominantly used for re-irradiation of patients who had been previously treated also with conventional radiotherapy (2DRT). This may indicate the importance of using advanced techniques in the setting of re-irradiation. In addition, we reported no event of urinary incontinence, which is a typical late event occurring in a significant percentage of patients undergoing salvage treatments after post-EBRT failure, in particular in those undergoing salvage prostatectomy [31, 32].Similar to the recent findings by Pasquier et al. [30], also in our study the biochemical control of disease post-PPR is correlated with the duration of the time interval between first EBRT and disease recurrence. Therefore, since our patient population was made up mostly of patients at high risk at initial diagnosis, the time interval to recurrence could identify a subset of patients with a better response to re-treatment.

Even more fascinating is the correlation between dose at first EBRT and local control after PPR. Assuming that the role of dose escalation in the improvement of all oncological outcomes including local control is a well-established data [2], an interpretation of this correlation as well as the finding that all prostatic recurrences occurred within the PPR treatment field [33], could be higher BED in the first treatment reduces the number of surviving clonogens, which could lead to a lower number of clonogens in the second treatment.

The role of the combination of ADT and radiotherapy in the patient setting evaluated in our study remains unclear. Because in our study the radiation re-treatment was considered as a possible alternative to systemic therapy, concomitant ADT was administered only to a minority of selected patients and for a short period of time. However, in our study, the role of concomitant ADT in disease control after PPR could not be determined. This is consistent with the data reported in most of the published literature, although the number of patients receiving concomitant ADT and the duration of the periods of ADT administration differs substantially among studies. We observed a trend for better biochemical control in patients undergoing combination treatment. A similar finding was also reported by Shipley et al. [34] and Carrie et al. [35] in patients treated with postoperative radiotherapy in association with Bicalutamide and Goserelin, respectively. The short follow-up time and the retrospective design of our study are two major shortcomings, which limit the evaluation of the role of ADT in these patients. A longer follow-up is necessary to determine which oncologic outcomes may potentially be improved by concomitant ADT. In addition, the statistical design of 
future studies should include the administration of ADT in this patient population and the comparison of outcomes by ADT in pre-determined subsets of patients.

A distinctive feature of our study is the use of the same radiation therapy technique and schedule in all patients at re-treatment. Although the radiation dose we employed in our patient population is in line with those reported in most papers, our patients received, at their first EBRT, a higher median radiation dose compared to patients treated in other studies. However, in spite of a high cumulative dose, the safety profile of PPR treatment remains excellent. JereczekFossa et al. [29] found a statistically significant improvement in a1-year biochemical progression-free survival rate and a 1 -year clinical progression-free survival rate when a BED $\geq 130$ Gy was prescribed. In our study, a BED of 151.7 Gy was prescribed to all patients with comparable 1-year outcomes and higher 2-years BFFS and 2-years CRFS suggesting that a dose escalation in this population could lead to improved outcomes.

To date, it is not yet clear what is the absolute and mutual role of the factors, which pose a limit to dose escalation in the re-treatment setting, namely: dose received by OARs and treatment volumes at primary EBRT, elapsed time, residual toxicity, re-irradiation volumes, and comorbidity. Therefore, dose-finding trials are necessary in this patient setting.

Although the rationale for PPR is to obtain the same or better oncological outcomes of re-irradiation of the entire gland but with an improvement in toxicity profile, its major limitation lies in the definition of the target volume. Even if we used fusion plans with MRI and/or [11C]-choline-PET, these imaging modalities have significant sensitivity limits that could result in an underestimation of the presence of tumor in the remaining prostate [36-39]. Nowadays, MRI is considered the best imaging modality to drive both diagnostic and therapeutic strategies for locally recurrent prostate cancer after primary radiotherapy [40]. Detection of recurrent prostate cancer is also feasible with choline PET/CT [41]: it should be noted that comparing data between choline PET and MRI in this setting of patients is still lacking. Recently, PSMA PET has been proposed in patients with BCR after primary treatment for prostate cancer. Although PET PSMA seems to have higher sensitivity to detect distant metastases and/or local relapse after radical prostatectomy with lower PSA values [42, 43], it remains unclear its superiority with respect to choline PET in the detection of local recurrence after primary EBRT with PSA values over Phoenix threshold [44]. The evaluation of the impact on the focal treatment of PSMA tracer could represent an object of future investigation.

Similarly, to most published investigations evaluating salvage re-irradiation for locally recurrent PCa [20-29], the small number of biopsies made represents a limitation of our study. The histological proof could in fact determine a potential modification of the volumes defined on the basis of imaging only [45]. However, we could assume that the imaging employed has been adequate in target definition since no intraprostatic out-field failure has been recorded. Additionally, other limitations of our study can be found in the retrospective nature of this report, in the limited number of patients treated, and in the relative shortness of the median follow-up. In particular, a longer follow-up is needed to obtain data on late toxicity, which is essential to determine the role of PPR.

Some caveats of our study need to be overcome to fulfill an effective long-time study. The aforementioned small number of biopsies made and the heterogeneity of diagnostic imaging at BCR represent crucial points to improve the patient selection with a larger number of biopsy-proven relapses and to homogenize treatment procedures (i.e. target delineation based on the same diagnostic tools). On these bases, a prospective long-term trial would confirm our results and provide more robust data on PPR.

Conversely, the homogeneity of our treatment modality both in the primary setting and in the recurrent disease, as well as the radiation technique employed can be regarded as points of strength of the present study.

There are some aspects of improvements in our study. Our PPR program has achieved efficacy results similar, or at least not inferior, to those reported in the literature but with an improved acute-toxicity profile. As for PPR feasibility, all patients completed the planned treatment, and the data on late toxicity, although not definitive, are very encouraging.

The statistical findings of our study seem to stress previously underestimated characteristics of recurrent prostate disease, such as the time to recurrence after primary EBRT and the cumulative dose in prostatic re-irradiation. Moreover, it must be emphasized that no out-field recurrences were reported, which confirms the validity of modern radiological and nuclear medicine techniques in the identification of recurrent disease and, consequently, in the definition of the radiation treatment volume.

The short-term results reported in this paper may provide the scientific community with updated information for a better understanding of PPR and for the implementation of this approach in patients with isolated intraprostatic recurrence.

To the best of our knowledge, this is the largest series exclusively dedicated to the PPR approach for isolated local PCa recurrence after primary EBRT. Even if retrospective, our study showed that PPR is a feasible and well-tolerated approach with a promising tumor control rate. These shortterm results may contribute to the growing evidence of PPR as a valid option in the context of focal salvage therapies for locally recurrent PCa. Long-term toxicity and the impact on disease control and patient survival will be evaluated with a longer follow-up time.

Acknowledgments: The authors would like to thank Daniela Michilin for her assistance in the final preparation of the manuscript. 


\section{References}

[1] MOHLER JL, ANTONARAKIS ES, ARMSTRONG AJ, D'AMICO AV, DAVIS BJ et al. Prostate Cancer, Version 2.2019, NCCN Clinical Practice Guidelines in Oncology. J Natl Compr Canc Netw 2019; 17: 479-505. https://doi. org/10.6004/jnccn.2019.0023

[2] KUBAN DA, LEVY LB, CHEUNG MR, LEE AK, CHOI S et al. Long-term failure patterns and survival in a randomized dose-escalation trial for prostate cancer. Who dies of disease? Int J Radiat Oncol Biol Phys 2011; 79: 1310-1317. https://doi.org/10.1016/j.ijrobp.2010.01.006

[3] ZELEFSKY MJ, KOLLMEIER M, COX B, FIDALEO A, SPERLING D et al. Improved clinical outcomes with highdose image guided radiotherapy compared with non-IGRT for the treatment of clinically localized prostate cancer. Int J Radiat Oncol Biol Phys 2012; 84: 125-129. https://doi. org/10.1016/j.ijrobp.2011.11.047.

[4] GOY BW, BURCHETTE R, SOPER MS, CHANG T, COSMATOS HA. Ten-Year Treatment Outcomes of Radical Prostatectomy Vs External Beam Radiation Therapy Vs Brachytherapy for 1503 Patients With Intermediate-risk Prostate Cancer. Urology 2020; 136: 180-189. https://doi. org/10.1016/j.urology.2019.09.040

[5] D’AMICO AV, WHITTINGTON R, MALKOWICZ SB, SCHULTZ D, BLANK K et al. Biochemical outcome after radical prostatectomy, external beam radiation therapy, or interstitial radiation therapy for clinically localized prostate cancer. JAMA 1998; 280: 969-974. https://doi.org/10.1001/ jama.280.11.969

[6] JANSEN BHE, VAN LEEUWEN PJ, WONDERGEM M, VAN DER SLUIS TM, NIEUWENHUIJZEN JA et al. Detection of Recurrent Prostate Cancer Using Prostate-specific Membrane Antigen Positron Emission Tomography in Patients not Meeting the Phoenix Criteria for Biochemical Recurrence After Curative Radiotherapy. Eur Urol Oncol 2020; S2588-9311(20)30009-2. https://doi.org/10.1016/j. euo.2020.01.002

[7] RAVEENTHIRAN S, YAXLEY J, GIANDUZZO T, KUA B, MCEWAN L et al. The use of 68Ga-PET/CT PSMA to determine patterns of disease for biochemically recurrent prostate cancer following primary radiotherapy. Prostate Cancer Prostatic Dis 2019; 22: 385-390. https://doi.org/10.1038/ s41391-019-0163-0

[8] MARRA G, VALERIO M, EMBERTON M, HEIDENREICH A, CROOK JM et al. Salvage Local Treatments After Focal Therapy for Prostate Cancer. Eur Urol Oncol 2019; 2: 526-538. https://doi.org/10.1016/j.euo.2019.03.008

[9] BATY M, CRÉHANGE G, PASQUIER D, PALARD X, DELEUZE A et al. Salvage re-irradiation for local prostate cancer recurrence after radiation therapy. For who? When? How? Cancer Radiother 2019; 23: 541-558. https://doi. org/10.1016/j.canrad.2019.07.125

[10] ALONGI F, DE BARI B, CAMPOSTRINI F, ARCANGELI S, MATEI DV et al. Salvage therapy of intraprostatic failure after radical external-beam radiotherapy for prostate cancer: a review. Crit Rev Oncol Hematol 2013; 88: 550-563. https:// doi.org/10.1016/j.critrevonc.2013.07.009
[11] ROACH M 3RD, HANKS G, THAMES H JR, SCHELLHAMMER P, SHIPLEY WU et al. Defining biochemical failure following radiotherapy with or without hormonal therapy in men with clinically localized prostate cancer: recommendations of the RTOG-ASTRO Phoenix Consensus Conference. Int J Radiat Oncol Biol Phys 2006; 65: 965-974. https://doi.org/10.1016/j.ijrobp.2006.04.029

[12] GAUR S, TURKBEY B. Prostate MR Imaging for Posttreatment Evaluation and Recurrence. Radiol Clin North Am 2018; 56: 263-275. https://doi.org/10.1016/j.rcl.2017.10.008

[13] WANG H, VEES H, MIRALBELL R, WISSMEYER M, STEINER C et al. 18F-fluorocholine PET-guided target volume delineation techniques for partial prostate re-irradiation in local recurrent prostate cancer. Radiother Oncol 2009; 93: 220-225. https://doi.org/10.1016/j.radonc.2009.08.037

[14] DINH CV, STEENBERGEN P, GHOBADI G, HEIJMINK SW, POS FJ et al. Magnetic resonance imaging for prostate cancer radiotherapy. Phys Med 2016; 32: 446-451. https:// doi.org/10.1016/j.ejmp.2016.01.484

[15] PANG EPP, KNIGHT K, FAN Q, TAN SXF, ANG KW et al. Analysis of intra-fraction prostate motion and derivation ofduration-dependent margins for radiotherapy using realtime 4D ultrasound. Phys Imaging Radiat Oncol 2018; 5: 102-107. https://doi.org/10.1016/j.phro.2018.03.008

[16] COX JD, STETZ J, PAJAK TF. Toxicity criteria of the Radiation Therapy Oncology Group (RTOG) and the European Organization for Research and Treatment of Cancer (EORTC). Int J Radiat Oncol Biol Phys 1995; 31: 1341-1346. https://doi.org/10.1016/0360-3016(95)00060-C

[17] KALBFLEISCH JD, PRENTICE RL. The Statistical Analysis of Failure Time Data. $2^{\text {nd }}$ ed. John Wiley \& Sons: Inc, 2002.

[18] D’AMICO AV, WHITTINGTON R, MALKOWICZ SB, SCHULTZ D, BLANK K et al. Biochemical outcome after radical prostatectomy, external beam radiation therapy, or interstitial radiation therapy for clinically localized prostate cancer. JAMA 1998; 280: 969-974. https://doi.org/10.1001/ jama.280.11.969

[19] VAN LEEUWEN CM, OEI AL, CREZEE J, BEL A, FRANKEN NAP et al. The alfa and beta of tumours: a review of parameters of the linear-quadratic model, derived from clinical radiotherapy studies. Radiat Oncol 2018; 13: 96. https://doi. org/10.1186/s13014-018-1040-Z

[20] JANORAY G, REYNAUD-BOUGNOUX A, RUFFIERLOUBIÈRE A, BERNADOU G, POINTREAU Y et al. Stereotactic body re-irradiation therapy for locally recurrent prostate cancer after external-beam radiation therapy: Initial report. Cancer Radiother 2016; 20: 275-281. https://doi. org/10.1016/j.canrad.2016.03.005

[21] MBEUTCHA A, CHAUVEINC L, BONDIAU PY, CHAND ME, DURAND $M$ et al. Salvage prostate re-irradiation using high-dose-rate brachytherapy or focal stereotactic body radiotherapy for local recurrence after definitive radiation therapy. Radiat Oncol 2017; 12: 49. https://doi.org/10.1186/ s13014-017-0789-9

[22] FULLER DB, WURZER J, SHIRAZI R, BRIDGE SS, LAW J et al. High-dose-rate stereotactic body radiation therapy for postradiation therapy locally recurrent prostatic carcinoma: Preliminary prostate-specific antigen response, disease-free survival, and toxicity assessment. Pract Radiat Oncol 2015; 5: e615-623. https://doi.org/10.1016/j.prro.2015.04.009 
[23] LOI M, DI CATALDO V, SIMONTACCHI G, DETTI B, BONOMO P et al. Robotic Stereotactic Retreatment for Biochemical Control in Previously Irradiated Patients Affected by Recurrent Prostate Cancer. Clin Oncol (R Coll Radiol) 2018; 30: 93-100. https://doi.org/10.1016/j.clon.2017.11.007

[24] D'AGOSTINO GR, DI BRINA L, MANCOSU P, FRANZESE C, IFTODE C et al. Reirradiation of Locally Recurrent Prostate Cancer With Volumetric Modulated Arc Therapy. Int J Radiat Oncol Biol Phys 2019; 104: 614-621. https://doi. org/10.1016/j.ijrobp.2019.02.041

[25] ZILLI T, BENZ E, DIPASQUALE G, ROUZAUD M, MIRALBELL R. Reirradiation of prostate cancer local failures after previous curative radiation therapy: long-term outcome and tolerance. Int J Radiat Oncol Biol Phys 2016; 96: 318322. https://doi.org/10.1016/j.ijrobp.2016.05.024

[26] SCHER N, BAUDUCEAU O, BOLLET M, LAMALLEM $\mathrm{H}, \mathrm{CHARAS} \mathrm{T}$ et al. Stereotactic prostate focal reirradiation therapy for local recurrence: preliminary results of Hartmann Oncology Radiotherapy Group. BJR Open 2019; 1: 20180027. https://doi.org/10.1259/bjro.20180027

[27] CUCCIA F, NICOSIA L, MAZZOLA R, FIGLIA V, GIAJLEVRA $\mathrm{N}$ et al. Linac-based SBRT as a feasible salvage option for local recurrences in previously irradiated prostate cancer. Strahlenther Onkol 2020; 196: 628-636. https://doi. org/10.1007/s00066-020-01628-6

[28] LEROY T, LACORNERIE T, BOGART E, NICKERS P, LARTIGAU E et al. Salvage robotic SBRT for local prostate cancer recurrence after radiotherapy: preliminary results of the Oscar Lambret Center. Radiat Oncol 2017; 12: 95. https:// doi.org/10.1186/s13014-017-0833-9

[29] JERECZEK-FOSSA BA, ROJAS DP, ZERINI D, FODOR C, VIOLA A et al. Reirradiation for isolated local recurrence of prostate cancer: Mono-institutional series of 64 patients treated with salvage stereotactic body radiotherapy (SBRT). Br J Radiol 2019; 92: 20180494. https://doi.org/10.1259/ bjr.20180494

[30] PASQUIER D, MARTINAGE G, JANORAY G, ROJAS DP, ZERINI D et al. Salvage Stereotactic Body Radiation Therapy for Local Prostate Cancer Recurrence After Radiation Therapy: A Retrospective Multicenter Study of the GETUG. Br J Radiol 2019; 92: 20180494. https://doi.org/10.1259/ bjr.20180494

[31] SANDERSON KM, PENSON DF, CAI J, GROSHEN S, STEIN JP et al. Salvage radical prostatectomy: quality of life outcomes and long-term oncological control of radiorecurrent prostate cancer. J Urol 2006; 176: 2025-2031; discussion 2031-2032. https://doi.org/10.1016/j.juro.2006.07.075

[32] LINARES ESPINÓS E, SÁNCHEZ-SALAS R, SIVARAMAN A, PEREZ-REGGETI JI, BARRET E et al. Minimally Invasive Salvage Prostatectomy After Primary Radiation or Ablation Treatment. Urology 2016; 94: 111-116. https://doi. org/10.1016/j.urology.2016.04.040

[33] PUCAR D, HRICAK H, SHUKLA-DAVE A, KUROIWA K, DROBNJAK $M$ et al. Clinically significant prostate cancer local recurrence after radiation therapy occurs at the site of primary tumor: magnetic resonance imaging and step-section pathology evidence. Int J Radiat Oncol Biol Phys 2007; 69: 62-69. https://doi.org/10.1016/j.ijrobp.2007.03.065
[34] SHIPLEY WU, SEIFERHELD W, LUKKA HR, MAJOR PP, HENEY NM et al. Radiation with or without Antiandrogen Therapy in Recurrent Prostate Cancer. N Engl J Med 2017; 376: 417-428. https://doi.org/10.1056/NEJMoa1607529

[35] CARRIE C, HASBINI A, DE LAROCHE G, RICHAUD P, GUERIF S et al. Salvage radiotherapy with or without shortterm hormone therapy for rising prostate-specific antigen concentration after radical prostatectomy (GETUG-AFU 16): a randomised, multicentre, open-label phase 3 trial. Lancet Oncol 2016; 17: 747-756. https://doi.org/10.1016/ S1470-2045(16)00111-X

[36] FANTI S, MINOZZI S, CASTELLUCCI P, BALDUZZI S, HERRMANN K et al. PET/CT with (11)C-choline for evaluation of prostate cancer patients with biochemical recurrence: meta-analysis and critical review of available data. Eur J Nucl Med Mol Imaging 2016; 43: 55-69. https://doi. org/10.1007/s00259-015-3202-7

[37] HAIDER MA, CHUNG P, SWEET J, TOI A, JHAVERI K et al. Dynamic contrast-enhanced magnetic resonance imaging for localization of recurrent prostate cancer after external beam radiotherapy. Int J Radiat Oncol Biol Phys 2008; 70: 425-430. https://doi.org/10.1016/j.ijrobp.2007.06.029

[38] ARUMAINAYAGAM N, KUMAAR S, AHMED HU, MOORE CM, PAYNE H et al. Accuracy of multiparametric magnetic resonance imaging in detecting recurrent prostate cancer after radiotherapy. BJU Int 2010; 106: 991-997. https://doi.org/10.1111/j.1464-410X.2010.09291.X

[39] ROY C, FOUDI F, CHARTON J, JUNG M, LANG H et al. Comparative sensitivities of functional MRI sequences in detection of local recurrence of prostate carcinoma after radical prostatectomy or external-beam radiotherapy. AJR Am J Roentgenol 2013; 200: W361-368. https://doi. org/10.2214/AJR.12.9106

[40] EAU GUIDELINES OFFICE. EAU Guidelines. Edn. presented at the EAU Annual Congress Amsterdam 2020. http://uroweb.org/guidelines/compilations-of-all-guidelines/

[41] CECI F, CASTELLUCCI P, GRAZIANI T, SCHIAVINA $\mathrm{R}$, BRUNOCILLA E et al. 11C-choline PET/CT detects the site of relapse in the majority of prostate cancer patients showing biochemical recurrence after EBRT. Eur J Nucl Med Mol Imaging 2014; 41: 878-886. https:/doi. org/10.1007/s00259-013-2655-9

[42] FAROLFI A, CECI F, CASTELLUCCI P, GRAZIANI T, SIEPE G et al. 68Ga-PSMA-11 PET/CT in prostate cancer patients with biochemical recurrence after radical prostatectomy and PSA $<0.5 \mathrm{ng} / \mathrm{ml}$. Efficacy and impact on treatment strategy. Eur J Nucl Med Mol Imaging 2019; 46: 11-19. https://doi.org/10.1007/s00259-018-4066-4

[43] PERERA M, PAPA N, ROBERTS M, WILLIAMS M, UDOVICICH C et al. Gallium-68 Prostate-specific Membrane Antigen Positron Emission Tomography in Advanced Prostate Cancer-Updated Diagnostic Utility, Sensitivity, Specificity, and Distribution of Prostate-specific Membrane Antigen-avid Lesions: A Systematic Review and Meta-analysis. Eur Urol 2020; 77: 403-417. https://doi.org/10.1016/j. eururo.2019.01.04 
[44] EVANS JD, JETHWA KR, OST P, WILLIAMS S, KWON ED et al. Prostate cancer-specific PET radiotracers: A review on the clinical utility in recurrent disease. Pract Radiat Oncol 2018; 8: 28-39. https://doi.org/10.1016/j.prro.2017.07.011
[45] KANOUN S, WALKER P, VRIGNEAUD JM, DEPARDON E, BARBIER $\mathrm{V}$ et al. 18F-Choline Positron Emission Tomography/Computed Tomography and Multiparametric Magnetic Resonance Imaging for the Detection of Early Local Recurrence of Prostate Cancer Initially Treated by Radiation Therapy: Comparison With Systematic 3-Dimensional Transperineal Mapping Biopsy. Int J Radiat Oncol Biol Phys 2017; 97: 986-994. https://doi.org/10.1016/j. ijrobp.2016.12.025 